\title{
A Time-Periodic Control Law for Satellite Magnetic Stabilization
}

\author{
Adrian-Mihail Stoica and Vasile Dragan
}

\begin{abstract}
The paper presents a design methodology for the automatic control system of a satellite with magnetic actuators taking into account the time periodic variation of the Earth's magnetic field. The design method is based on using an optimal $\mathrm{H}_{2}$ controller with periodic coefficients. These coefficients depend on the solution of a specific system of coupled Riccati type equations. An iterative numerical algorithm to solve such system is also presented. The proposed design procedure is illustrated by a case study for a CubSat in which the benefits of using a time-periodic controller are emphasized.
\end{abstract}

Index Terms $-H_{2}$ optimal control, satellite stabilization, system of coupled Riccati equations, time periodic control law.

\section{INTRODUCTION}

The satellites control problems received a considerable attention since the early space applications from five decades ago. The interest for the design of attitude control system (ACS) design is motivated by the continuous diversification of space missions in which more and more companies, research entities and universities are involved. A special attention has been devoted in recent years to the so-called CubSats with small dimensions and low mass (cubs with $10 \mathrm{~cm}$ sides and the mass less than $1.33 \mathrm{~kg}$ ). Such nano-satellites may be successfully used for data acquisition, Earth observation and commercial services. Some of the main CubSats' advantages include their low price and reduced launching costs. In spite of their small dimensions of a CubSat the design of their ACS is a challenging problem from the perspective of a control engineer due to the inherent constraints imposed by the limited onboard hardware resources. This is why a trade-off between the performances level and the control laws complexity must be taken into account. Among the numerous solutions adopted for the ACS design one mentions the classical proportional-derivative (PD) control algorithms (see e.g. [1], [2]), optimal linear-quadratic-Gaussian(LQG) based control ([3], [4]) and more recently, advanced control techniques based on norm minimization approaches ([4], [5]). By contrast with the most of the above mentioned solutions which are time invariant control laws, in the present paper a design procedure

Manuscript received March 1, 2017; revised May 10, 2017. This paper has been supported by MEN-UEFISCDI, Program Partnerships, Projects PN-II-PT-PCCA- 2013-4-1349.

A.-M. Stoica is with the University "Politehnica" of Bucharest, Faculty of Aerospace Engineering, Str. Polizu, No. 1, Ro-011061, Bucharest, Romania (e-mail: adrian.stoica@upb.ro).

V. Dragan is with the Institute of Mathematics "Simion Stoilow" of the Romanian Academy, Bucharest, Romania (e-mail: vasile.dragan@imar.ro). providing a controller with time-periodic coefficients is proposed. It is assumed that the CubSat has a low Earth orbit (LEO) and its stabilization is performed using electromagnetic actuators which magnetic fields interact with magnetic field of the Earth. Due to the periodic evolution of the satellite around the Earth, the components of the Earth magnetic field are time-periodic and therefore it is a natural option to design a controller with time-periodic coefficients. The chosen design procedure is based on the minimization of the $\mathrm{H}_{2}$ norm of the mapping from the sensors noises approximated by random white noise signals, to the satellite angular rate. ACS design based on the $\mathrm{H}_{2}$ norm minimization problem have been also considered in [6] and [7] where state-feedback control laws have been determined. By contrast with these results, in the present paper an output feedback $\mathrm{H}_{2}$ controller is used avoiding thus the estimation of unmeasured stated.

The paper is organized as follows: the next section presents some preliminaries concerning the time-periodic model of the Earth's magnetic field together with the design models of the CubSat. Then, in the the third section, the analytical expressions of the time-periodic controller are given. Numerical simulations based on the proposed periodic controller are presented and discussed in the fourth section. The paper ends with some concluding remarks. The following notations are used throughout the paper: by " $T$ " the transposed of a matrix is denoted, $I_{n \times n}$ is the unit matrix of dimension $n \times n, P>0(P<0)$ stands for a symmetric matrix $P \in \mathbb{R}^{n \times n}$ positive (negative) definite.

\section{PReliminaries AND Design Models}

The satellite's attitude dynamics actuated by magnetic control torques is described using the Euler's differential equation ([1], [2])

$$
\dot{\omega} t=-\omega t \times \mathbf{I} \omega t+T_{m a g} t
$$

where $\omega \in \mathbb{R}^{3}$ denotes the angular velocity expressed in body frame, $\mathbf{I} \in \mathbb{R}^{3 \times 3}$ stands for the the moment of inertia and $T_{m a g} \in \mathbb{R}^{3}$ is the control input which components are the control torques.

The attitude kinematics parameterized using quaternions is given by

$$
\dot{q} t=\frac{1}{2} \Omega t q t
$$


where $q \in \mathbb{R}^{4}$ is the quaternion vector having unitary Euclidian norm and

$$
\Omega t=\left[\begin{array}{cccc}
0 & \omega_{x} & -\omega_{y} & \omega_{z} \\
-\omega_{z} & 0 & \omega_{x} & \omega_{y} \\
\omega_{y} & -\omega_{x} & 0 & \omega_{z} \\
-\omega_{x} & -\omega_{y} & -\omega_{z} & 0
\end{array}\right],
$$

$\omega_{x}, \omega_{y}, \omega_{z}$ denoting the components of the angular rate vector. Usually the angular speed components from the above equations are different since the ones arising in (1) are expressed with respect to the Earth-centered inertial (ECI) frame, while the ones from (2) correspond to the orbital frame called Local Vertical Local Horizontal (LVLH) frame. To simplify the design models bellow it was assumed that the angular speed expressed with respect to the two reference frames are the same, which assumption depends on the mission specific. The control vector has the expression

$$
T_{m a g}=m \times b
$$

where $m \in \mathbb{R}^{3}$ consists of the three magnetic dipoles generated by the actuator coils and $b=\left[\begin{array}{lll}b_{x} & b_{y} & b_{z}\end{array}\right]^{T}$ includes the components of the Earth's magnetic field. In the above equation the cross product $m \times b$ will be expressed in the equivalent form

$$
m \times b=\mathbf{B} b m
$$

where by definition,

$$
\text { B } b=\left[\begin{array}{ccc}
0 & b_{z} & -b_{y} \\
-b_{z} & 0 & b_{x} \\
b_{y} & -b_{x} & 0
\end{array}\right] \text {. }
$$

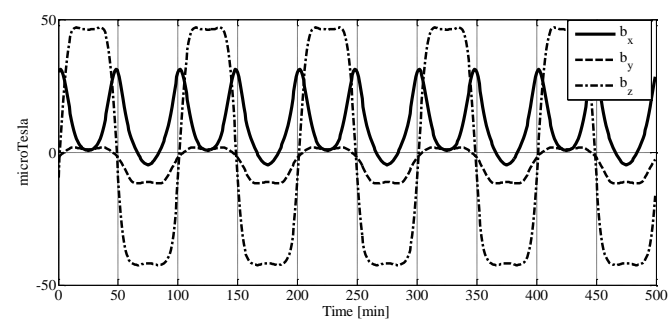

Fig. 1. The Earth's magnetic components variation over five periods.

The components $b_{x}, b_{y}, b_{z}$ of the Earth's magnetic field depend on the geographic coordinates of the point where they are measured and they be determined using the International Geomagnetic Reference Field (IGRF) models depending on the latitude, longitude and the altitude of the the measurement point. For a polar LEO of a CubSat at $500 \mathrm{~km}$ altitude, the components of the Earth magnetic field have the following variation during five orbits, for an orbital period of $100 \mathrm{sec}$, obtained the IGRF model implemented in the MATLAB package.

Based on the least squares method, the following Fourier type approximations have been obtained for the above components of the Earth's magnetic field

$$
\begin{aligned}
& \mathrm{b}_{x} t=10.0232+3.6848 \cdot \sin \left(\frac{2 \pi}{100} \mathrm{t}\right)+0.4388 \cdot \cos \left(\frac{2 \pi}{100} \mathrm{t}\right) \\
& -0.0001 \cdot \sin \left(\frac{4 \pi}{100} \mathrm{t}\right)+15.2696 \cdot \cos \left(\frac{4 \pi}{100} \mathrm{t}\right) ; \\
& \mathrm{b}_{y} t=-4.1344+7.5875 \cdot \sin \left(\frac{2 \pi}{100} \mathrm{t}\right)+0.0570 \cdot \cos \left(\frac{2 \pi}{100} \mathrm{t}\right) \\
& -0.0000 \cdot \sin \left(\frac{4 \pi}{100} \mathrm{t}\right)+1.4810 \cdot \cos \left(\frac{4 \pi}{100} \mathrm{t}\right) ; \\
& \mathrm{b}_{z} \rightarrow-0.7554+53.7496 \cdot \sin \left(\frac{2 \pi}{100} \mathrm{t}\right)-0.3448 \cdot \cos \left(\frac{2 \pi}{100} \mathrm{t}\right) \\
& +0.0000 \cdot \sin \left(\frac{4 \pi}{100} \mathrm{t}\right)-5.0603 \cdot \cos \left(\frac{4 \pi}{100} \mathrm{t}\right) .
\end{aligned}
$$

Since the design approach for the CubSat's ACS is linear, the linear approximations of the dynamic and kinematics equations (1) and (2) have been determined as follows:

$$
A=\left[\begin{array}{ll}
\frac{\partial \dot{q}}{\partial q} & \frac{\partial \dot{q}}{\partial \omega} \\
\frac{\partial \dot{\omega}}{\partial q} & \frac{\partial \dot{\omega}}{\partial \omega}
\end{array}\right], B t=\left[\begin{array}{c}
0_{4 \times 3} \\
\mathbf{I}^{-1}
\end{array}\right] \mathbf{B} b t
$$

where $\partial \dot{q} / \partial q, \ldots, \partial \dot{\omega} / \partial \omega$ denote the derivatives of the right sides from equations (2) and (1), respectively. For $I_{x}=0.00528 \mathrm{~kg} \cdot \mathrm{m}^{2}, I_{y}=0.00513 \mathrm{~kg} \cdot \mathrm{m}^{2}$ and

$I_{z}=0.00202 \mathrm{~kg} \cdot \mathrm{m}^{2}$, the following linearized model around the equilibrium conditions $q=\begin{array}{lllll}0 & 0 & 0 & 1^{T} \text { and }\end{array}$ $\omega=0 \quad 0 \quad 0^{T}$ has been obtained

$$
\dot{x} t=A x t+B t u t
$$

with

$$
\begin{aligned}
& A=\left[\begin{array}{cc}
0_{3 \times 4} & \frac{1}{2} I_{3 \times 3} \\
0_{4 \times 4} & 0_{4 \times 3}
\end{array}\right], \\
& B t=\left[\begin{array}{ccc}
189.3939 & 0 & 0 \\
0 & 194.9318 & 0 \\
0 & 0 & 495.0495
\end{array}\right] \text { B } b t,
\end{aligned}
$$

where $x:=\left[\begin{array}{ll}q^{T} & \omega^{T}\end{array}\right]^{T}$ and $u t=m t$.

\section{Time-PERIODIC $H_{2}$ OPtimal CONTROL System}

Consider the following discrete-time linear system denoted 
by $G$

$$
\begin{aligned}
& x t+1=A t x t+B t u t+B_{\xi} t \xi t \\
& y t=C t x t+D_{\xi} t \xi t \\
& z t=C_{z} t x t+D_{z} t u t, t=0,1, \ldots
\end{aligned}
$$

where the matrices $A t, B t, B_{\xi} t, C t, D_{\xi} t, C_{z} t$ and $D_{z} t$ are matrices with $\theta$-periodic elements, $\theta \in \mathbb{N}$, namely $A t+\theta=A t$, etc. It is assumed that the exogenous input $\xi t_{t \geq 0}$ is a sequence of random vectors satisfying the assumptions that $E[\xi t]=0$ and $E\left[\begin{array}{llll}\xi & t & \xi^{T} & t\end{array}\right]=I$, where $E \cdot$ denotes the mathematical expectation. In (9), y $t$ denotes the measurements vector and $z t$ is a quality output. The aim is to design a discrete-time periodic controller denoted by $G_{c}$ with the state space equations

$$
\begin{aligned}
& x_{c} t+1=A_{c} t x_{c} t+B_{c} t u_{c} t \\
& y_{c} t=C_{c} t x_{c} t, t=0,1, \ldots, \theta
\end{aligned}
$$

such that the closed loop system obtained when coupling the controller (10) to the system (9) making $u_{c} t=y t$ and $u t=y_{c} t$, is stable and its $H_{2}$ norm from the $\xi t$ to the quality output $z t$ is minimal. Definition of the $H_{2}$ for a stable periodic system for the more general case when the system is corrupted with multiplicative noise and Markovian jumps may be found in [8] where explicit formulae for the optimal $\mathrm{H}_{2}$ controller are also derived. In the case when the multiplicative noise and the Markovian jumps are missing as in the case of the periodic system (10), these formulae become

$$
\begin{aligned}
& A_{c} t=A t+B t F t+K t C t \\
& B_{c} t=-K t \\
& C_{c} t=F t
\end{aligned}
$$

with

$$
\begin{aligned}
F t=- & B^{T} t X t+1 B t+D_{\xi}^{T} t D_{\xi} t^{-1} \\
& \times B^{T} t X t+1 A t+D_{\xi} t^{T} D_{\xi} t \\
K t=- & A t Y t C^{T} t+B_{\xi} t D_{\xi}^{T} t \\
& \times C t Y t C^{T} t+D_{\xi} t D_{\xi}^{T} t^{-1},
\end{aligned}
$$

$t=0,1, \ldots, \theta-1$, where $X t$ and $Y t$ are the solutions of the algebraic Riccati type equations

$$
\begin{aligned}
X t= & A^{T} \\
& t X t+1 A t+C_{z}^{T} t C_{z} t \\
& \times A^{T} t X t+1 B t+C_{z}^{T} t D_{z} t \\
& \times B^{T} t X t+1 B t+D_{z}^{T} t D_{z} t^{-1} \\
& \times A^{T} t X t+1 B t+C_{z}^{T} t D_{z} t^{T}
\end{aligned}
$$

and

$$
\begin{aligned}
Y t+1= & A t Y t A^{T} t+B_{\xi} t B_{\xi}^{T} t \\
- & A t Y t C^{T} t+B_{\xi} t D_{\xi}^{T} t \\
& \times C t Y t C^{T} t+D_{\xi} t D_{\xi}^{T} t^{-1} \\
& \times A t Y t C^{T} t+B_{\xi} t D_{\xi}^{T} t^{T},
\end{aligned}
$$

respectively, $t=0,1, \ldots, \theta-1$

In order to solve the systems of Riccati type equations (13) and (14) the iterative procedure described in [8], may be used.

\section{NUMERICAL RESULTS}

The design procedure described in the previous section has been used to determine a discrete-time periodic ACS for the satellite which design model has been presented in Section II. To this end one must firstly determine the discretized system of the continuous-time system (7). The discretization time used for this case study was $T_{d}=20 \mathrm{~min}$ and therefore, since the period was assumed $T=100 \mathrm{~min}$, it results that $\theta=5$ for the formulae presented in the previous section. To this end the following well-known expressions of the discretized system have been used

$$
A_{d}=e^{A T_{d}} \text { and } B_{d}=\int_{0}^{T_{d}} e^{A T_{d}-\tau} B d \tau .
$$

Therefore in the formulae for the discrete-time periodic controller given in Section III, the matrices $A$ and $B$ must be replaced by $A_{d}$ and $B_{d}$, respectively. Since in our case study $A$ is constant, from the above expression it follows that $A_{d}$ is also constant and only $B_{d}$ is time periodic with the period $\theta=5$. The following quality output have been considered for this application

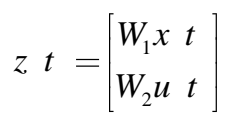

where $W_{1} \in \mathbb{R}^{7 \times 7}, W_{2} \in \mathbb{R}^{3 \times 3}, \quad W_{1}>0$ and $W_{2}>0$ are weighting matrices chosen depending on the imposed admissible magnitude of the the states and the control variables. Thus it follows that 


$$
C_{z}=\left[\begin{array}{c}
W_{1} \\
0_{3 \times 7}
\end{array}\right] \text { and } D_{z}=\left[\begin{array}{c}
0_{3 \times 7} \\
W_{2}
\end{array}\right] \text {. }
$$

The exogenous input consists of the measurement noise of the inertial measurement unit (IMU) providing the angular rate and therefore

$$
B_{\xi}=0_{7 \times 3}, C=0_{3 \times 4} \quad I_{3 \times 3}, D_{\xi}=10^{-3} \cdot I_{3 \times 3}
$$

Let us notice that since in this application only the matrix $B_{d} t$ is periodic time-varying, the system (14) simply reduces to a discrete-time algebraic Riccati equation which can be solved using usual numerical procedures as the ones implemented in MATLAB. As concerns the system (13) it consists of $\theta$ coupled algebraic Riccati-type equations which can be numerically solved using the following iterative procedure ([8]).

Step 1. Determine a stabilizing feedback gain $F_{0} t, t=0, \ldots, \theta-1$ for the system

$$
x t+1=A_{d} x t+B_{d} t u t .
$$

Such stabilizing gain may be obtained as $F t=W t Z^{-1} t$ where $W t$ and $Z t>0$ satisfy the system of linear matrix inequalities (LMIs)

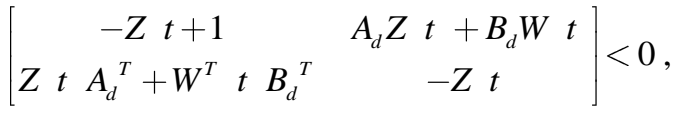

Set $k=0$.

$$
t=0, \ldots, \theta-1 \text {. }
$$

Step 2. For $k \leftarrow k+1$, compute the solution $X_{k} t>0, t=0, \ldots, \theta-1$ of the system of LMI's

$$
\begin{aligned}
& A_{d}+B_{d} t F_{k-1} t^{T} X_{k} t+1 \quad A_{d}+B_{d} t F_{k-1} t \\
& -X_{k} t+C_{z}+D_{z} F_{k-1} t^{T} C_{z}+D_{z} F_{k-1} t \quad+\delta I_{7 \times 7} \leq 0
\end{aligned}
$$

with $\delta>0$ arbitrarily chosen and

$$
\begin{aligned}
F_{k} t & =-B_{d}{ }^{T} t X_{k} t+1 B_{d}+D_{z}^{T} D_{z}{ }^{-} \\
& \times B_{d}{ }^{T} t X_{k} t+1 A_{d}+D_{z}^{T} C_{z},
\end{aligned}
$$

$t=0, \ldots, \theta-1$;

Repeat Step 2 until

$\max \left\|X_{k} \quad 0-X_{k-1} 0\right\|, \ldots,\left\|X_{k} \theta-1-X_{k-1} \theta-1\right\|<\varepsilon$ where $\varepsilon>0$ is a small scalar arbitrarily chosen.

Using the above numerical procedure for $W_{1}=I_{7 \times 7}$ and $W_{2}=10 \cdot I_{3 \times 3}$ one obtained the following matrices for the realization of the time-periodic optimal $\mathrm{H}_{2}$ controller of form (11):

$\mathrm{A}_{c} 0=$
$\left[\begin{array}{ccccccc}0.9863 & -0.0829 & -0.0273 & 0 & -1.8292 & -54.4142 & -35.4992 \\ -0.0824 & 0.1130 & 0.0044 & 0 & -52.3518 & -562.7608 & 3.8242 \\ 0.0482 & 0.0338 & 0.6871 & 0 & 36.0633 & 8.2647 & -391.6650 \\ 0 & 0 & 0 & 0.99 & 0 & 0 & 0 \\ 0 & -0.0003 & -0.0001 & 0 & -0.0061 & -0.1814 & -0.1183 \\ -0.0003 & -0.0029 & 0 & 0 & -0.1745 & -1.8759 & 0.0127 \\ 0.0002 & 0.0001 & -0.0010 & 0 & 0.1202 & 0.0275 & -1.3055\end{array}\right]$

$A_{c} 1=$
$\left[\begin{array}{ccccccc}0.5977 & -0.0431 & -0.1759 & 0 & -428.4995 & -29.3054 & -235.3142 \\ -0.0116 & 0.1400 & -0.0828 & 0 & -8.4778 & -564.1263 & -103.7862 \\ 0.0257 & 0.0782 & 1.0084 & 0 & 27.7454 & 51.9606 & 24.0430 \\ 0 & 0 & 0 & 0.99 & 0 & 0 & 0 \\ -0.0013 & -0.0001 & -0.0006 & 0 & -1.4283 & -0.0977 & -0.7844 \\ 0 & -0.0028 & -0.0003 & 0 & -0.0283 & -1.8804 & -0.3460 \\ 0.0001 & 0.0003 & 0.0001 & 0 & 0.0925 & 0.1732 & 0.0801\end{array}\right]$

$A_{c} 2=$

$\left[\begin{array}{ccccccc}0.8218 & -0.0904 & 0.1258 & 0 & -189.997 & -20.8255 & 146.7527 \\ -0.1432 & 0.2126 & 0.0509 & 0 & -92.438 & -537.0429 & -17.6156 \\ 0.2554 & 0.1817 & 0.8026 & 0 & 284.016 & 64.5000 & -213.7347 \\ 0 & 0 & 0 & 0.99 & 0 & 0 & 0 \\ -0.0006 & -0.0003 & 0.0004 & 0 & -0.633 & -0.0694 & 0.4892 \\ -0.0005 & -0.0026 & 0.0002 & 0 & -0.308 & -1.7901 & -0.0587 \\ 0.0009 & 0.0006 & -0.0006 & 0 & 0.9467 & 0.2150 & -0.7124\end{array}\right]$

A. $3=$

$\left[\begin{array}{ccccccc}0.8304 & -0.1198 & -0.2601 & 0 & -148.475 & -165.0634 & -287.4227 \\ 0.0476 & 0.3346 & 0.2698 & 0 & 15.227 & -498.0179 & 81.8786 \\ -0.1810 & 0.2912 & 0.5762 & 0 & -150.423 & 150.9362 & -323.5708 \\ 0 & 0 & 0 & 0.99 & 0 & 0 & 0 \\ -0.0005 & -0.0004 & -0.0009 & 0 & -0.495 & -0.5502 & -0.9581 \\ 0.0002 & -0.0022 & -.0009 & 0 & 0.051 & -1.6601 & 0.2729 \\ -0.0006 & 0.0010 & -0.0014 & 0 & -0.501 & 0.5031 & -1.0786\end{array}\right]$

$A_{c} 4=$

$\left[\begin{array}{ccccccc}0.6601 & 0.0042 & 0.0451 & 0 & -389.3546 & 19.713 & 41.5356 \\ 0.0538 & 0.5759 & 0.5277 & 0 & 90.5030 & -265.599 & 399.4690 \\ 0.0669 & 0.2739 & 0.6253 & 0 & 60.9722 & 170.376 & -278.3934 \\ 0 & 0 & 0 & 0.99 & 0 & 0 & 0 \\ -0.0011 & 0 & 0.0002 & 0 & -1.2978 & 0.066 & 0.1385 \\ 0.0002 & -0.0014 & 0.0018 & 0 & 0.3017 & -0.885 & 1.3316 \\ 0.0002 & 0.0009 & -0.0012 & 0 & 0.2032 & 0.568 & -0.9280\end{array}\right]$

$B_{c} 0=B_{c} 1=B_{c} 2=B_{c} 3=B_{c} 4=\left[\begin{array}{ccc}600 \cdot I_{3 \times 3} \\ 0 & 0 & 0 \\ 0.99 \cdot I_{3 \times 3}\end{array}\right]$

$C_{c} \quad 0=\left[\begin{array}{ccccccc}0 & 0.0001 & 0 & 0 & -0.0072 & -0.0701 & 0.0085 \\ 0 & 0 & 0.0001 & 0 & -0.0071 & -0.0053 & 0.0845 \\ 0 & -0.0005 & 0 & 0 & -0.0273 & -0.2949 & 0.0001\end{array}\right]$

$C_{c} \quad 1=\left[\begin{array}{ccccccc}0 & 0.0002 & 0 & 0 & 0.0023 & 0.1478 & 0.0272 \\ -0.0001 & 0 & 0 & 0 & -0.1155 & -0.0080 & -0.0634 \\ 0 & 0 & 0 & 0 & 0.0040 & -0.0083 & 0.0016\end{array}\right]$

$C_{c} 2=\left[\begin{array}{ccccccc}0.0001 & 0.0003 & 0 & 0 & 0.0349 & 0.1973 & 0.0057 \\ -0.0001 & 0 & 0.0001 & 0 & -0.0947 & -0.0131 & 0.0727 \\ 0 & -0.0002 & 0 & 0 & -0.0172 & -0.1102 & -0.0050\end{array}\right]$

$C_{c} 3=\left[\begin{array}{ccccccc}0 & -0.0002 & 0.0001 & 0 & 0.0105 & -0.1823 & 0.0401 \\ 0.0001 & 0 & 0.0001 & 0 & 0.0620 & 0.0527 & 0.1217 \\ 0 & -0.0001 & 0 & 0 & -0.0113 & -0.0791 & -0.0150\end{array}\right]$ 


$$
C_{c} 4=\left[\begin{array}{ccccccc}
0 & -0.0001 & 0.0002 & 0 & 0.0231 & -0.0778 & 0.1177 \\
0.0001 & 0 & 0 & 0 & 0.1109 & -0.0034 & -0.0153 \\
0 & 0 & 0 & 0 & -0.0318 & 0.0101 & -0.0100
\end{array}\right]
$$

For the initial condition

$$
x 0=0 \begin{array}{lllllll} 
& 0 & 0 & 1 & 0 & 0 & \pi / 10^{T}
\end{array}
$$

The following time responses of the angular rates and of the controls have been obtained with the above time-periodic controller
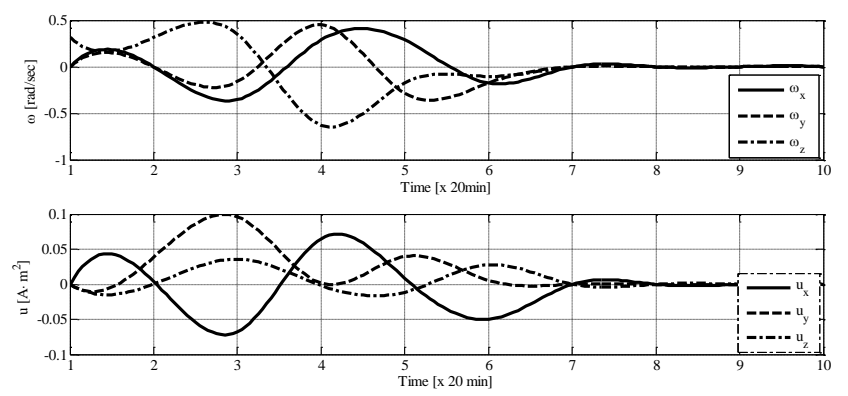

Fig. 2. Time responses of angular rates and controls obtained with the time-periodic controller

Further, the case when using a unique $\mathrm{H}_{2}$ controller has been considered. For the controller with the realization $A_{c} 5, B_{c} 5, C_{c} 5$, the following time responses have been obtained:
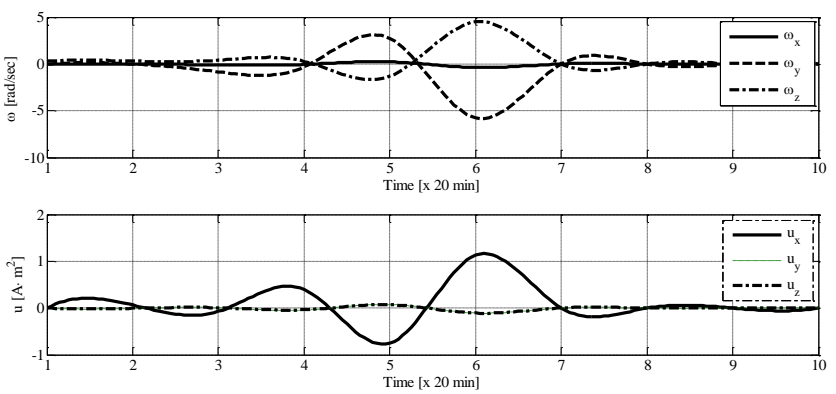

Fig. 3. Time responses of angular rates and controls obtained with the constant controller $A_{c} 5, B_{c} 5, C_{c} 5$.

Comparing the time responses above one can see that for the time constant controller the responses amplitude are significantly higher than in the case when the optimal time-periodic controller is used. Note that the settling time is also larger for time constant controller.

\section{CONCLUSIONS}

A design methodology for the ACS of a CubSat magnetically actuated have been developed taking into account the time-periodic variation of the Earth's magnetic field. The design procedure is based on the minimization of the $H_{2}$ norm induced from the measurement white noises to a quality output including the state and the control variables. It was shown that the optimal $H_{2}$ controller is time varying with time-periodic coefficients. The numerical analyses indicate better performances of the time-periodic controller concerning the attenuation properties and the settling time, with respect to the case when ACS with constant parameters are used.

\section{REFERENCES}

[1] J. Wertz, Spacecraft Attitude Determination and Control, Dordrecht: D. Reidel, 1978.

[2] M. Sidi, Spacecraft Dynamics and Control, Cambridge, 1997.

[3] A. Tewari, Atmospheric and Space Flight Dynamics, Birkhäuser, 2007.

[4] B. Wie, Space Vehicles Dynamics and Control, AIAA, Inc., 1998.

[5] M. Lovera,"Periodic H-infinity attitude control for satellites with magnetic actuators," in Porc. 3rd IFAC Symposium on Robust Control Design, Prague, Czech Republic, 2000

[6] J.-F. Trégouët, D. Arzelier, D. Peaucelle, Y. Ehihara, C. Pittet, and A. Facolz, "Periodic $\mathrm{H}_{2}$ synthesis for spacecraft attitude control with magnetorquers and reaction wheels," in Porc. 50th IEEE Conference on Decision and Control and European Control Conference, Orlando, FL, USA, 12-15 December 2011.

[7] R. Wisniewski and J. Stoustrup, "Periodic $\mathrm{H}_{2}$ synthesis for spacecraft attitude determination and control with a vector magnetometer and magnetorquers," Journal of Guidance, Control, and Dynamics, vol. 27, no. 5, pp. 874-881, 2004.

[8] V. Dragan, T. Morozan, and A.-M. Stoica, "Output-based $H_{2}$ controllers for a class of discrete-time stochastic linear systems with periodic coefficients," International Journal of Robust and Nonlinear Control, vol. 25, no. 13, pp. 1897-1926, 2015.

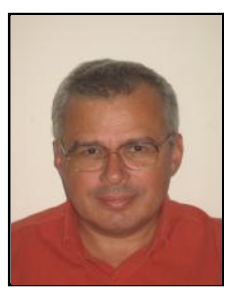

Adrian-Mihail Stoica was born in Bucharest, Romania on November 30, 1954. He graduated the Faculty of Automatic Control of University "Politehnica" of Bucharest in 1979. He received the $\mathrm{Ph} . \mathrm{D}$. degree in control engineering from the University "Politehnica" of Bucharest in 1993. Currently, he is a professor at Faculty of Aerospace Engineering of University "Politehnica" of Bucharest. He is author or co-author of five monographs and more than one hundred fifty refereed journal and conferences papers. His research interests include automatic flight control systems, robust control theory, estimation and filtering, numerical algorithms. Prof. Stoica is member of the Technical Committee on Aerospace of International Federation of Automatic Control (IFAC) and member of American Mathematical Society (AMS).

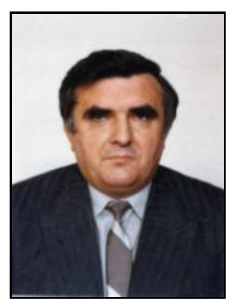

Vasile Dragan was born in Valea Bolvasnita, Caras-Severin, Romania on 2 February 1950. Since 1965 till 1969 he studied at "Traian Lalescu" High School, from Mehadia. He received Ph.D. degree in 1979 from University of Bucharest, Romania. Now he is senior researcher to Institute of Mathematics of Romania Academy, in Department of Differential Equations. His research interests are in qualitative theory of differential equations mainly in singular perturbation techniques, stability theory and asymptotic expansions. Also, his current research interests include control theory, robust control for deterministic and stochastic systems. The Romanian Academy

awarded him "Gheorghe Lazar" prize in 1994. He has authored or co-authored over 200 journal articles and Conference publications. Dr. Dragan is member of American Mathematical Society (AMS). 